\title{
Global Burden of Disease Study 2013 reports that disability caused by CKD is increasing worldwide
}

Chronic kidney disease (CKD) is an increasing cause of disability worldwide, according to new data from the Global Burden of Disease Study (GBD) 2013. "This paper expands on our prior mortality study - which showed that CKD was the noncommunicable cause of death that increased the most in the past 23 years when assessed as a mean rank of

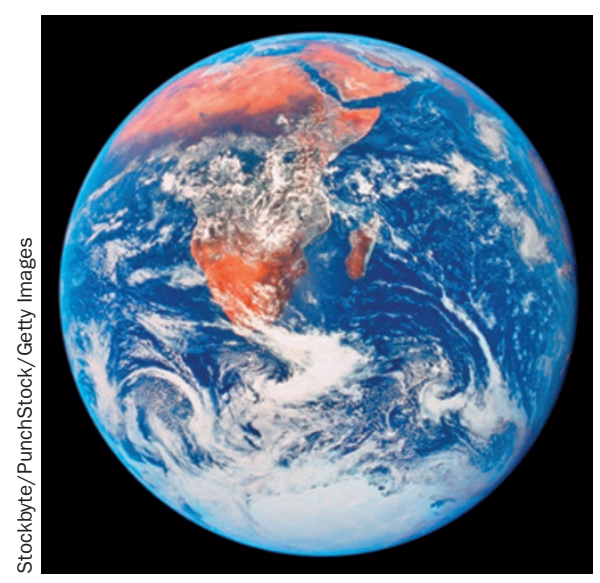

global years of life lost-by demonstrating that CKD is also an increasing cause of years lived with disability (YLDs)," says GBD 2013 collaborator Alberto Ortiz.

The new analysis, which includes data from 35,620 distinct sources in 188 countries, reports that YLDs for all causes increased by $42.3 \%$ from 537.6 million in 1990 to 764.8 million in 2013. By contrast, the age standardized rate of YLD for all causes decreased by $3.9 \%$ during this period. Overall, only $4.3 \%$ of the global population had no burden of disease or injury sequelae in 2013.

For CKD, YLDs increased by $49.5 \%$ to 12.3 million and the age-standardized YLD rate decreased by $2.8 \%$ between 1990 and 2013. Trends in age-standardized YLD rates differed, however, between specific causes of CKD; age-standardized rates for CKD due to hypertension and CKD due to glomerulonephritis decreased by $22.4 \%$ and $6.9 \%$, respectively, whereas those for $\mathrm{CKD}$ due to diabetes mellitus and CKD due to other causes increased by $10.6 \%$ and $6.9 \%$, respectively. Moreover, the number of cases of CKD due to diabetes and of CKD due to other causes increased by more than $50 \%$ during the study period.

The collaborators note that "in all countries, the share of disability in total [disease] burden is increasing because of ageing populations and a slower decline in disability rates compared to the decline in mortality." They hope that the findings of GBD 2013 and future studies will be used to guide health policy as well as the provision and financing of health services.

\section{Ellen F. Carney}

Original article Global Burden of Disease Study 2013 Collaborators. Global, regional, and national incidence, prevalence, and years lived with disability for 301 acute and chronic diseases and injuries in 188 countries, 1990-2013: a systematic analysis for the Global Burden of Disease Study 2013. Lancet doi:10.1016/S01406736(15)60692-4 